Jorge Vitório Pereira

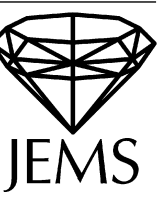

\title{
The characteristic variety of a generic foliation
}

Received December 9, 2009

Abstract. We confirm a conjecture of Bernstein-Lunts which predicts that the characteristic variety of a generic polynomial vector field has no homogeneous involutive subvarieties besides the zero section and subvarieties of fibers over singular points.

Keywords. Characteristic foliation, invariant variety, $\mathcal{D}$-modules

\section{Introduction}

\subsection{Foliations}

Let $\mathcal{F}$ be a one-dimensional singular holomorphic foliation on a smooth projective variety $X$. The characteristic variety $\operatorname{ch}(\mathcal{F})$ of $\mathcal{F}$ is the irreducible subvariety of $E\left(T^{*} X\right)$, the total space of the cotangent bundle of $X$, with fiber over a non-singular point $x \in X_{0}=$ $X \backslash \operatorname{sing}(\mathcal{F})$ equal to the 1 -forms at $x$ which vanish on $T_{x} \mathcal{F}$. More succinctly, if $N^{*} \mathcal{F}$ is the conormal sheaf of $\mathcal{F}$ then its restriction at $X_{0}$ is a vector subbundle of $T^{*} X_{0}$ and we can write

$$
\operatorname{ch}(\mathcal{F})=\overline{E\left(N^{*} \mathcal{F}_{\mid X_{0}}\right)}
$$

where the closure is taken in $E\left(T^{*} X\right) \supset E\left(T^{*} X_{0}\right)$.

Clearly $\operatorname{ch}(\mathcal{F})$ is a hypersurface of $E\left(T^{*} X\right)$. If $\omega$ is the non-degenerate 2-form which induces the canonical symplectic structure on $T^{*} X$ then its restriction to $\operatorname{ch}(\mathcal{F})$ induces a one-dimensional foliation $\mathcal{F}^{(1)}$ on (the smooth locus of) $\operatorname{ch}(\mathcal{F})$ which will be called the first prolongation of $\mathcal{F}$.

In this work we are interested in the subvarieties of $\operatorname{ch}(\mathcal{F})$ invariant under $\mathcal{F}^{(1)}$ when $\mathcal{F}$ is sufficiently general. For no matter which $\mathcal{F}$ there is always at least one subvariety of $\operatorname{ch}(\mathcal{F})$ invariant under $\mathcal{F}^{(1)}$ : the zero section of $T^{*} X$. If the singular set of $\mathcal{F}$ is non-empty but of dimension zero then the fibers over it, and some subvarieties of these fibers, are also left invariant by $\mathcal{F}^{(1)}$.

J. V. Pereira: IMPA, Estrada Dona Castorina, 110, 22460-320, Rio de Janeiro, RJ, Brazil; e-mail: jvp@impa.br 
We will say that $\operatorname{ch}(\mathcal{F})$ is a quasi-minimal characteristic variety if (a) $\mathcal{F}$ has isolated singularities; and (b) every irreducible homogeneous (on the fibers of $\operatorname{ch}(\mathcal{F}) \rightarrow X$ ) subvariety of $\operatorname{ch}(\mathcal{F})$ left invariant by $\mathcal{F}^{(1)}$ is either the zero section, or a subvariety of a fiber over the singular set of $\mathcal{F}$, or the whole $\operatorname{ch}(\mathcal{F})$.

Theorem 1. Let $X$ be a smooth projective variety, $\mathcal{L}$ an ample line bundle over it, and $k \gg 0$ a sufficiently large integer. If $\mathcal{F} \in \mathbb{P} H^{0}\left(X, T X \otimes \mathcal{L}^{\otimes k}\right)$ is a very generic foliation then $\operatorname{ch}(\mathcal{F})$ is a quasi-minimal characteristic variety.

In the statement of the theorem above and throughout, by a very generic point of a given variety we mean a point outside a countable union of Zariski closed subvarieties. The expression generic point will be reserved to points outside a finite union of Zariski closed subvarieties.

Although Theorem 1 can be thought of as a natural development of Jouanolou's Theorem and its subsequent generalizations (see [6] and references therein), it is motivated by a problem coming from the representation theory of Weyl algebras that we briefly review below.

\subsection{Weyl algebras}

Let $A_{n}$ be the $n$-th Weyl algebra over $\mathbb{C}$, that is, $A_{n}$ is the algebra of $\mathbb{C}$-linear differential operators on the polynomial ring $\mathbb{C}\left[x_{1}, \ldots, x_{n}\right]$. A basic invariant of an irreducible $A_{n}$ module $M$ is its Gelfand-Kirillov dimension $G K \operatorname{dim} M$. By Bernstein's work [2] this invariant is subject to the inequality $G K \operatorname{dim} M \geq n$ and equality holds true for important classes of irreducible $A_{n}$-modules. If $G K \operatorname{dim} M=n$ then $M$ is, by definition, a holonomic $A_{n}$-module.

For some time, some believed that every irreducible $A_{n}$-module $M$ was holonomic. In 1985 Stafford came up with examples of $A_{n}$-modules of particularly simple form and having Gelfand-Kirillov dimension equal to $2 n-1$. His examples are of the form $A_{n} / I A_{n}$ where $I$ is a principal left ideal generated by an element of the form $\xi+f$ where $\xi$ is a polynomial vector field and $f$ is a polynomial (see [11]). For those not familiar with the Gelfand-Kirillov dimension it is useful to remark that when $I$ is a principal maximal left ideal then $G K \operatorname{dim} A_{n} / I A_{n}=2 n-1$, and the search for examples of non-holonomic $A_{n}$-modules can be reduced to searching for principal maximal left ideals of $A_{n}$.

Stafford's examples are explicit and his arguments are purely algebraic. In [3], Bernstein and Lunts present two geometrically oriented approaches to constructing principal maximal left ideals of $A_{n}$, and implement them for the second Weyl algebra. In rough terms, their strategy relies on the the study of a natural foliation defined on the characteristic varieties of the module. More specifically, they relate the maximality of the ideal to the non-existence of proper invariant subvarieties of this foliation. To define a characteristic variety for an $A_{n}$-module, a filtration of $A_{n}$ has to be fixed and their two approaches are determined by the choice of two different filtrations.

In the first approach they look at the Bernstein filtration of $A_{n}$, whose $i$-th piece $A_{n}^{i}$ consists of polynomials in $\left\{x_{1}, \ldots, x_{n}, \partial_{x_{1}}, \ldots, \partial_{x_{n}}\right\}$ of degree at most $i$. The correspond- 
ing symbol maps are

$$
\sigma_{k}: A_{n}^{k} \rightarrow A_{n}^{k} / A_{n}^{k-1} \simeq \mathbb{C}_{k}\left[x_{1}, \ldots, x_{n}, y_{1}, \ldots, y_{n}\right]
$$

They proved that if $n=2, k \geq 4$ and $P \in \mathbb{C}_{k}\left[x_{1}, \ldots, x_{n}, y_{1}, \ldots, y_{n}\right]$ is a very generic polynomial then each operator $d \in A_{n}^{k}$ satisfying $\sigma_{k}(d)=P$ generates a maximal left ideal of $A_{n}^{k}$. Still under the assumption that $k \geq 4$, Lunts extends the above result to arbitrary $n \geq 2$ in [8]. For $k=3$ and $n \geq 2$ the same has been proved by McCune [9]. All these results, in contrast with Stafford's, do not exhibit explicit examples of nonholonomic $A_{n}$-modules but instead prove that they are generic in the above sense. For an algorithm to produce explicit examples of the above form for $n=2$ and its implementation see [1].

In their second approach, Bernstein and Lunts look at the standard filtration of $A_{n}$. Now the $i$-th piece corresponds to differential operators of order $\leq i$. If $\xi$ is a polynomial vector field, $f$ a polynomial and $I=\langle\xi+f\rangle$ then the characteristic variety of $A_{n} / I A_{n}$ coincides with the characteristic variety of the foliation $\mathcal{F}_{\xi}$ as defined in the previous section. If $\mathcal{F}_{\xi}$ has a quasi-minimal characteristic variety then according to [3, Proposition 6] there exists $f \in \mathbb{C}\left[x_{1}, \ldots, x_{n}\right]$ for which $I=\langle\xi+f\rangle$ is maximal. While they do show that a generic $\xi$ of degree $\geq 2$ on $\mathbb{C}^{2}$ has this property, they leave the general case as a conjecture (see [3, §4.2]).

Conjecture (Bernstein-Lunts). Let $n \geq 2$ and $\xi$ be a very generic polynomial vector field on $\mathbb{C}^{n}$ with coefficients of degree $\geq 2$. Then $\operatorname{ch}\left(\mathcal{F}_{\xi}\right)$ is a quasi-minimal characteristic variety.

The three-dimensional case of the conjecture has been proved recently by Coutinho [5]. In this paper we will settle the general case.

Theorem 2. Bernstein-Lunts' conjecture holds true.

Even when specialized to $n=3$, our proof is very different from the one of Coutinho.

\section{Characteristic varieties and prolongations}

\subsection{Characteristic variety}

Let $X$ be a quasi-projective manifold and $\mathcal{F}$ be a foliation on $X$ with cotangent bundle $\mathcal{L}$, that is, $\mathcal{F}=[\xi] \in \mathbb{P} H^{0}(X, T X \otimes \mathcal{L})$ with the representative $\xi$ having no divisorial components in its singular set. As in the introduction set $X_{0}=X \backslash \operatorname{sing}(\mathcal{F})$.

Contraction with the twisted vector field $\xi$ determines a morphism of $\mathcal{O}_{X}$-modules

$$
T^{*} X \rightarrow \mathcal{L}
$$

whose kernel is $N^{*} \mathcal{F}$, the conormal sheaf of $\mathcal{F}$. At points $x \in X_{0}$ the sheaf $N^{*} \mathcal{F}$ is clearly locally free, but it is not locally free in general. For example it is never locally free at an isolated singularity of $\mathcal{F}$ as one can promptly verify. Nevertheless, the restriction of $N^{*} \mathcal{F}$ 
to $X_{0}$ determines a subbundle of $T^{*} X_{0}$ of corank one. As mentioned in the introduction, $\operatorname{ch}(\mathcal{F})$ is defined as the closure in $E\left(T^{*} X\right)$ of $E\left(N^{*} \mathcal{F}_{\mid X_{0}}\right)$. We will use $\pi$ to denote the natural projection $\pi: E\left(T^{*} X\right) \rightarrow X$ as well as its restriction $\pi: \operatorname{ch}(\mathcal{F}) \rightarrow X$.

If $\left(x_{1}, \ldots, x_{n}\right)$ are local coordinates on an open subset $U \subset X$ then the vector fields $\left\{\partial_{x_{i}}=\partial / \partial x_{i}\right\}$ can be thought of as linear coordinates on $T^{*} U$ : the value of $\partial_{x_{i}}$ at a 1 -form $\omega \in T^{*} U$ is given by the contraction $\omega\left(\partial_{x_{i}}\right)$. Thus, if we set $y_{i}=\partial_{x_{i}}$ then $\left(x_{1}, \ldots, x_{n}, y_{1}, \ldots, y_{n}\right)$ are global coordinate functions for $T^{*} U$. In particular, if $\xi=$ $\sum a_{i} \partial_{x_{i}}$ then

$$
\operatorname{ch}(\mathcal{F})_{\mid \pi^{-1}(U)}=\left\{\sum a_{i} y_{i}=0\right\} .
$$

The singular set of $\operatorname{ch}(\mathcal{F})$ is contained in $\pi^{-1}(\operatorname{sing}(\mathcal{F}))$ and contains $\pi^{-1}(\operatorname{sing}(\mathcal{F}))$ $\cap X$, where $X$ sits inside $E\left(T^{*} X\right)$ as the zero section. Thus, unless $\mathcal{F}$ is a smooth foliation, $\operatorname{ch}(\mathcal{F})$ is always singular. It follows promptly from the above local expression of $\operatorname{ch}(\mathcal{F})$ that its singular points away from the zero section and over a fiber $\pi^{-1}(p)$ are the 1-forms at $T_{p}^{*} X$ which annihilate the image of $D \xi(p)$. Thus, if the singular scheme of $\mathcal{F}$ is reduced and of dimension zero then $\operatorname{ch}(\mathcal{F})$ is smooth away from the zero section.

\subsection{Prolongation}

Recall that $T^{*} X$ is endowed with a canonical symplectic structure which, in the above local coordinates, is induced by the 2 -form

$$
\Omega=\sum d x_{i} \wedge d y_{i}
$$

If $F$ is a holomorphic function on (an open subset of) $T^{*} X$ then the hamiltonian of $F$ is by definition the vector field $\xi_{F}$ determined by the formula

$$
d F(\cdot)=\Omega\left(\xi_{F}, \cdot\right)
$$

Notice that the vector field $\xi_{F}$ is tangent to the hypersurface determined by $F$ since $\xi_{F}(F)=0$. Leibniz's rule implies that $\xi_{u F}=u \xi_{F}+F \xi_{u}$. Consequently, the restriction of the direction field determined by $\xi_{F}$ to $\{F=0\}$ is the same as the one of that determined by $\xi_{u F}$ for an arbitrary unit $u$. Therefore, the symplectic structure determines a one-dimensional foliation on any reduced and irreducible hypersurface $H \subset T^{*} X$ : one has just to factor out possible divisorial components of the singular set of $\xi_{F \mid H}$ to end up with a foliation on $H$, usually called in the literature the characteristic foliation of $H$. When $H=\operatorname{ch}(\mathcal{F}) \subset T^{*} X$ is the characteristic variety of a foliation $\mathcal{F}$ on $X$ we will denote its characteristic foliation by $\mathcal{F}^{(1)}$ and call it the first prolongation of $\mathcal{F}$.

If $U \subset X$ is an open set with coordinates as in $\S 2.1$ and $\xi=\sum a_{i} \partial_{x_{i}}$ is a vector field inducing $\mathcal{F}$ on $U$ then the vector field

$$
\hat{\xi}=\sum_{i=1}^{n} a_{i} \partial_{x_{i}}-\sum_{i, j=1}^{n}\left(\partial_{x_{j}} a_{i}\right) y_{i} \partial_{y_{j}}
$$

is the hamiltonian vector field of $\sum a_{i} y_{i}$, and hence defines the prolongation of $\mathcal{F}_{\mid U}$. 


\section{Warm-up: Proof of Theorem 1 in dimension three}

In this section we present a proof of Theorem 1 in dimension three. We believe this will make the general case easier to understand.

\subsection{Making sense of the $\mathcal{F}^{(1)}$-invariance}

We start by clarifying the meaning of $\mathcal{F}^{(1)}$-invariance. The first result is well-known and holds in arbitrary dimension.

Lemma 3.1. If $Y \subset \operatorname{ch}(\mathcal{F})$ is $\mathcal{F}^{(1)}$-invariant then $\pi(Y)$ is $\mathcal{F}$-invariant.

Proof. If $p$ is a smooth point of $\operatorname{ch}(\mathcal{F})$ then $(2.1)$ makes it clear that $\pi$ sends $T_{p} \mathcal{F}^{(1)}$ into $T_{\pi}(p) \mathcal{F}$, and that the restriction of $\mathcal{F}^{(1)}$ to the zero section is nothing other than $\mathcal{F}$. Together these two facts promptly imply the lemma.

Our next result holds only in dimension three, and it is the lack of a direct analogue in higher dimensions which will make the proof in the general case more involved.

Proposition 3.2. Suppose $n=3$ and let $Y \subsetneq \operatorname{ch}(\mathcal{F})$ be a homogeneous irreducible subvariety with dominant projection to $X$. If $Y^{-}$is $\mathcal{F}^{(1)}$-invariant then $\mathcal{F}$ is tangent to a codimension one web $\mathcal{W}_{Y}$ on $X$.

Proof. Since we are in dimension three, over the smooth locus of $\mathcal{F}, \operatorname{ch}(\mathcal{F})$ is a rank two vector subbundle of $\Omega_{X}^{1}$. A subvariety $Y$ as in the statement determines $k$ distinct lines on $N^{*} \mathcal{F}_{x}$ for generic points $x \in X$. Therefore $Y$ can be seen as the graph of a rational section $\varpi$ of $\operatorname{Sym}^{k} \Omega_{X}^{1}$. Moreover, the foliation $\mathcal{F}$ is tangent to the multi-distribution determined by $\varpi$. Notice that so far, we have not used the $\mathcal{F}^{(1)}$-invariance of $Y$ : we just explored the fact that $Y$ is contained in $\operatorname{ch}(\mathcal{F})$.

It remains to prove the integrability of the multi-distribution determined by $\varpi$. To do so we can place ourselves in a neighborhood of a point $x \in X$ where $\varpi$ is holomorphic and equal to the product of $k$ pairwise distinct 1 -forms, say $\omega_{1}, \ldots, \omega_{k}$, and $\mathcal{F}$ is smooth. Choose a local coordinate system $\left(x_{1}, \ldots, x_{n}\right)$ where $\mathcal{F}$ is induced by the vector field $\xi=\partial_{x_{1}}$. Hence $\mathcal{F}^{(1)}$ is still induced by $\partial_{x_{1}}$ now seen as a vector field on the total space of $N^{*} \mathcal{F}$.

If $\omega$ is any of the 1 -forms $\left\{\omega_{i}\right\}_{i \in\{1, \ldots, k\}}$ then $\omega=a d x_{2}+b d x_{3}$ for suitable holomorphic functions $a, b$. Notice that $\omega$ is integrable if and only if the quotient $a / b$ does not depend on $x_{1}$. Finally, the $\mathcal{F}^{(1)}$-invariance of $Y$ ensures that $a / b$ is constant along the orbits of $\hat{\xi}$ and thus $\omega$ is integrable and so is the multi-distribution induced by $\varpi$.

\subsection{Invariant subvarieties from singular points}

Proposition 3.3. Let $\mathcal{F}$ be a foliation on $X$ a smooth projective variety of dimension three. Suppose $\mathcal{F}$ is tangent to a codimension one web $\mathcal{W}$. If $p \in \operatorname{sing}(\mathcal{F})$ is an isolated singularity then there exists an irreducible $\mathcal{F}$-invariant subvariety $Y \subsetneq X$ of positive dimension containing $p$. 
Proof. Suppose $\mathcal{W}$ is a $k$-web with $k \geq 1$. If $k \geq 2$, let $\Delta(\mathcal{W}) \subset X$ be the discriminant of the web $\mathcal{W}$. By definition, $\Delta(\mathcal{W})$ is the set where $\mathcal{W}$ is not the product of $k$ pairwise transverse foliations. The proof of Proposition 3.2 tells us that on a neighborhood of a smooth point of $\mathcal{F}$, the web $\mathcal{W}$ is induced by a $k$-symmetric 1 -form $\varphi=\sum a_{i j} d x_{2}^{i} d x_{3}^{j}$. Thus $\Delta(\mathcal{W})$ is defined as the hypersurface cut out by the discriminant of $\varpi$, seen as a binary form in the variables $d x_{2}, d x_{3}$. Notice that $\Delta(\mathcal{W})$ is an $\mathcal{F}$-invariant hypersurface.

If $p$ belongs to $\Delta(\mathcal{W})$ we are done. Otherwise $\mathcal{W}$, in a neighborhood of $p$, can be written as the superposition of $k$ foliations, $\mathcal{W}=\mathcal{G}_{1} \otimes \cdots \otimes \mathcal{G}_{k}$. So consider one foliation $\mathcal{G}$ of codimension one in a neighborhood of $p$ and suppose that $\mathcal{F}$ is tangent to it.

Let $\xi$ be a holomorphic vector field inducing $\mathcal{F}$ and $\omega$ be a holomorphic 1-form inducing $\mathcal{G}$, both defined on a neighborhood of $p$ and without divisorial components in their zero sets. Since $\mathcal{F}$ has an isolated singularity at $p$, so does $\xi$. Consequently, $\omega(\xi)=0$ implies that $\omega$ is also singular at $p$. At this point we can use an argument laid down by Cerveau in $[4$, p. 46] that we now recall. As $\xi$ has isolated singularities we can apply the de Rham-Saito Lemma to ensure the existence of another vector field $\zeta$ such that $\omega=i_{\xi} i_{\zeta} d x \wedge d y \wedge d z$. Therefore the zero set of $\omega$ is formed by the minors of a $3 \times 2$ matrix and must be of codimension at least two. But if $\mathcal{G}$ is one of the foliations $\mathcal{G}_{i}$ then $\operatorname{sing}(\mathcal{G})$ is algebraic, and is the desired $\mathcal{F}$-invariant variety.

\subsection{Conclusion of the proof}

To conclude the proof of Theorem 1 in dimension three we will make use of the following generalization of Jouanolou's Theorem proved in [6] (see also [8, Theorem 2] for the very same statement on projective spaces).

Theorem 3.4. Let $X$ be a smooth projective variety, $\mathcal{L}$ be an ample line bundle over it, and $k \gg 0$ be a sufficiently large integer. If $\mathcal{F} \in \mathbb{P} H^{0}\left(X, T X \otimes \mathcal{L}^{\otimes k}\right)$ is a very generic foliation then, besides $X$ itself, the only subvarieties left invariant by $\mathcal{F}$ are its singular points.

Let $\mathcal{F} \in \mathbb{P} H^{0}\left(X, T X \otimes \mathcal{L}^{\otimes k}\right)$ be a very generic foliation without invariant subvarieties. As its singular set has cardinality given by the top Chern class of $T X \otimes \mathcal{L}^{\otimes k}$, and this number is positive for $k \gg 0$, the singular set of $\mathcal{F}$ is non-empty. Moreover we can assume the existence of an isolated singularity $p \in \operatorname{sing}(\mathcal{F})$ (see for instance [6, Proposition 2.4]).

If the characteristic variety of $\mathcal{F}$ is not quasi-minimal then Proposition 3.2 implies that $\mathcal{F}$ is tangent to a codimension one web $\mathcal{W}$. Proposition 3.3 in its turn implies that $\mathcal{F}$ has an invariant subvariety through $p$. This contradicts Theorem 3.4 and concludes the proof of Theorem 1 in dimension three.

\subsection{Obstructions to generalize}

To generalize the argument above to deal with the general case one has to circumvent the following obstructions: 
(1) Proposition 3.2 does not generalize because irreducible components of $\operatorname{ch}(\mathcal{F})$ which are homogeneous and dominate the base $X$ are no longer graphs of multi-distributions as happens in the three-dimensional case; and

(2) Proposition 3.3 does not generalize since (multi)-distributions with infinitesimal automorphisms are not necessarily integrable.

To accomplish that we will take advantage of the structure of generic foliation singularities combined with the following reinterpretation of Theorem 3.4.

Theorem 3.5. Let $X$ be a smooth projective variety, $\mathcal{L}$ an ample line bundle over it, and $k \gg 0$ a sufficiently large integer. If $\mathcal{F} \in \mathbb{P} H^{0}\left(X, T X \otimes \mathcal{L}^{\otimes k}\right)$ is a very generic foliation then every leaf of $\mathcal{F}$ is Zariski dense.

\section{Prolongation versus holonomy}

In this section $\mathcal{F}$ will be a smooth foliation of dimension one on a complex manifold $X$.

\subsection{Holonomy}

To each leaf $L$ of $\mathcal{F}$, once a point $p \in L$ and a germ $(\Sigma, p)$ of smooth hypersurface transverse to $\mathcal{F}$ are fixed, one can associate an (anti)-representation

$$
\operatorname{hol}(L): \pi_{1}(L, p) \rightarrow \operatorname{Diff}(\Sigma, p)
$$

as follows. Given a closed path $\gamma$ contained in $L$ and centered at $p$ one defines a germ of diffeomorphism $h_{\gamma} \in \operatorname{Diff}(\Sigma, p)$ such that $h_{\gamma}(x)$ is the end point of a lift of $\gamma$ to the leaf of $\mathcal{F}$ through $x$. The result does not depend on the choices involved in the process and is completely determined by the class of $\gamma$ in $\pi_{1}(L, p)$. Thus one can set $h o l(L)(\gamma)=h_{\gamma}$. It is an anti-representation since $h_{\gamma_{1} \cdot \gamma_{2}}=h_{\gamma_{2}} \circ h_{\gamma_{1}}$.

Of course, one can also consider the linear holonomy of $L$ which is just the antirepresentation

$$
\operatorname{Dhol}(L): \pi_{1}(L, p) \rightarrow G L\left(T_{p} \Sigma\right), \quad[\gamma] \mapsto D h_{\gamma}(p) .
$$

Since this is an anti-representation of $\pi_{1}(L)$ to a general linear group, it is natural to wonder if there is a natural connection on a natural vector bundle over $L$ inducing it. It is indeed the case, and even better, there is a partial connection along the tangent bundle $T \mathcal{F}$ of $\mathcal{F}$ on the normal bundle $N \mathcal{F}$ whose monodromy along the leaves of $\mathcal{F}$ is equivalent to the linear holonomy.

\subsection{Bott's partial connection}

Let $\rho: T X \rightarrow N \mathcal{F}$ be the natural projection. Of course ker $\rho=T \mathcal{F}$. Bott's partial connection is defined as follows:

$$
\nabla: T \mathcal{F} \rightarrow \operatorname{Hom}(N \mathcal{F}, N \mathcal{F}) \simeq N^{*} \mathcal{F} \otimes N \mathcal{F}, \quad \xi \mapsto\{\vartheta \mapsto \rho([\hat{\vartheta}, \xi])\},
$$

where $\hat{\vartheta}$ stands for an arbitrary lift of $\vartheta$ to $T X$. The involutiveness of $T \mathcal{F}$ implies that $\rho([\hat{\vartheta}, \xi])$ does not depend on the choice of the lift, and ensures that $\nabla$ is well defined. 
Let us now proceed to write explicitly the restriction of $\nabla$ to a leaf $L$ of $\mathcal{F}$. We will work in local coordinates $\left(x_{1}, \ldots, x_{n}\right)$ and will assume that $L=\left\{x_{2}=\cdots=x_{n}=0\right\}$. Since $L$ is invariant under $\mathcal{F}$, we can write a vector field $\xi$ generating $T \mathcal{F}$ in the form

$$
\xi=a(x) \partial_{x_{1}}+\sum_{i=2}^{n} \sum_{j=2}^{n} a_{i j}(x) x_{i} \partial_{x_{j}}
$$

Notice that the vector fields $\partial_{x_{2}}, \ldots, \partial_{x_{n}}$ can be interpreted as a basis of $N \mathcal{F}$. Thus

$$
\nabla(\xi)\left(\partial_{x_{i}}\right)=\rho\left(\partial_{x_{i}} a(x) \partial_{x_{1}}+\sum_{i=2}^{n} \sum_{j=2}^{n}\left(\partial_{x_{i}} a_{i j}(x)\right) x_{i} \partial_{x_{j}}+\sum_{j=2}^{n} a_{i j}(x) \partial_{x_{j}}\right) .
$$

Hence, the induced connection $\nabla_{\mid L}: T L \rightarrow N^{*} L \otimes N L$ is

$$
\begin{aligned}
\nabla_{\mid L}(\xi) & =\sum_{i=1}^{n-1} \sum_{j=2}^{n} a_{i j}\left(x_{1}, 0\right) d x_{i} \otimes \partial_{x_{j}} \\
& =\left(d x_{2}, \ldots, d x_{n}\right) \cdot A\left(x_{1}, 0\right) \cdot\left(\partial_{x_{2}}, \ldots, \partial_{x_{n}}\right)^{T} .
\end{aligned}
$$

\subsection{Comparison with the prolongation}

In order to compare with Bott's connection, let us now write down the restriction to $\pi^{-1}(L)$ of the lift of $\xi$ to $E\left(N^{*} \mathcal{F}\right)$. We will use the same system of coordinates used in Section 2.1, where $y_{i}=\partial_{x_{i}}$. Since in these coordinates $\pi^{-1}(L)=\left\{y_{1}=x_{2}=\cdots=\right.$ $\left.x_{n}=0\right\}$, we can write

$$
\hat{\xi}_{\mid \pi^{-1}(L)}=a\left(x_{1}, 0\right) \partial_{x_{1}}-\sum_{i, j=2}^{n}\left(a_{j i}\left(x_{1}, 0\right)\right) y_{i} \partial_{y_{j}},
$$

which in matrix form is

$$
\hat{\xi}_{\mid \pi^{-1}(L)}=a\left(x_{1}, 0\right) \partial_{x_{1}}-\left(y_{2}, \ldots, y_{n}\right) \cdot A^{T}\left(x_{1}, 0\right) \cdot\left(\partial_{y_{2}}, \ldots, \partial_{y_{n}}\right)^{T}
$$

with $A\left(x_{1}, 0\right)$ being the same matrix as in (4.1). It is then clear that in these coordinates, the leaves of $\mathcal{F}^{(1)}$ restricted to $\pi^{-1}(L)$ are flat sections of the connection on $N^{*} L$ having connection matrix $-A^{T}$, where $A$ is the connection matrix on $\nabla_{\mid L}$. We have thus proved the following

Proposition 4.1. The leaves of $\mathcal{F}^{(1)}$ are flat sections of the partial connection dual to Bott's partial connection.

\section{From invariant subvarieties to multi-distributions}

\subsection{Non-resonant singularities}

Let $\mathcal{F}$ be a germ of one-dimensional foliation on $\left(\mathbb{C}^{n}, 0\right)$. Suppose that it has an isolated singularity at the origin. Suppose also that the linear part $D \xi(0)$ of a vector field $\xi$ inducing $\mathcal{F}$ is invertible and its eigenvalues $\lambda_{1}, \ldots, \lambda_{n}$ generate a $\mathbb{Z}$-module of rank $n$. We will say that a singularity of this form is a non-resonant singularity. 
Lemma 5.1. There exist $n$ germs of $\mathcal{F}$-invariant smooth curves $\gamma_{i}:(\mathbb{C}, 0) \rightarrow\left(\mathbb{C}^{n}, 0\right)$ with tangents at zero determined by the eigenvectors of $D \xi(0)$.

Proof. The Hadamard-Perron theorem for holomorphic flows [7, Chapter 2, Section 7] ensures the existence of a pair of invariant manifolds intersecting transversely at the origin and such that the restriction of the vector field to each of them has a non-resonant singularity in the Poincaré domain (Section 5 loc. cit.). Poincaré's normalization theorem (loc. cit.) implies that the corresponding restrictions of $\xi$ are analytically linearizable. Since separatrices of the restrictions of $\xi$ are also separatrices of $\xi$, the lemma follows.

The linear holonomy along a positive oriented path around the origin contained in $\gamma_{i}(\mathbb{C}, 0)$ is induced by a linearizable matrix $A_{i} \in G L(n-1, \mathbb{C})$ with eigenvalues $\left\{\exp \left(2 \pi i \lambda_{j} / \lambda_{i}\right)\right\}_{j \neq i}$. Moreover, the $\mathbb{Z}$-independence of the eigenvalues implies that the Zariski closure of the subgroup of $G L\left(\mathbb{C}^{n-1}\right)$ generated by $A_{i}$ is a maximal torus $\simeq\left(\mathbb{C}^{*}\right)^{n-1}$.

\subsection{Singularities and the holonomy of separatrices}

Together with Proposition 6.2, the proposition below will replace Proposition 3.2 in the proof of the general case of Theorem 1. It guarantees that invariant subvarieties of $\mathcal{F}^{(1)}$ correspond to multi-distributions tangent to $\mathcal{F}$ as soon as $\mathcal{F}$ has non-resonant singularities.

Proposition 5.2. Let $\mathcal{F}$ be a foliation on a smooth projective variety $X$ and let $Y \subsetneq$ $\operatorname{ch}(\mathcal{F})$ be an irreducible subvariety with dominant projection to $X$ distinct from the zero section. Suppose $\mathcal{F}$ has non-resonant singularity $p$ and that at least one of its separatrices is Zariski dense. If $Y$ is $\mathcal{F}^{(1)}$-invariant then the fiber of $Y$ over a generic point of $X$ is a finite union of linear spaces of the same dimension. Consequently, $\mathcal{F}$ is tangent to a multi-distribution of codimension $q=\operatorname{dim} Y-\operatorname{dim} X \leq \operatorname{dim} X-2$.

Proof. First consider a point $p_{0} \in X$ in the Zariski dense separatrix through $p$, and let $L$ be the leaf of $\mathcal{F}$ through it. The fiber $V$ of $E\left(N^{*} \mathcal{F}\right) \simeq \operatorname{ch}(\mathcal{F}) \rightarrow X$ over $p$ is a vector space of dimension $n-1$. The intersection of $Y$ with $V$ is a subvariety of $V$ invariant under the image $G \subset G L(V)$ of the representation $\pi_{1}(L) \rightarrow G L(V)$ dual to the linear holonomy of $L$. Since $V \cap Y$ is algebraic, not only $G$ but also its Zariski closure leaves $V \cap Y$ invariant. By hypothesis, $\bar{G} \simeq\left(\mathbb{C}^{*}\right)^{n-1}$ is a maximal torus in $G L(V)$. Consequently, $V \cap Y$ is a finite union of linear spaces for an arbitrary $p \in L$. To be a finite union of linear subspaces is clearly a Zariski closed condition. Thus the same will hold true for the fibers of $Y$ over points in the Zariski closure of $L$ which is, by assumption, equal to $X$.

\section{From multi-distributions to invariant subvarieties}

We now proceed to establish the result which will replace Proposition 3.3. We start with a simple lemma. 
Lemma 6.1. Let $\omega \in \Omega^{q}=\Omega^{q}\left(\mathbb{C}^{n}\right) \otimes \mathbb{C}\left[\left[x_{1}, \ldots, x_{n}\right]\right]$ be a formal $q$-form. If $\omega$ is invariant under the natural $\left(\mathbb{C}^{*}\right)^{n}$-action on $\mathbb{C}^{n}$ then

$$
\omega=f \cdot\left(\sum_{I \in\{1, \ldots, n\}^{q}} \lambda_{I} \frac{d x_{I}}{x_{I}}\right)
$$

where $f \in \mathbb{C}\left[\left[x_{1}, \ldots, x_{n}\right]\right], \lambda_{I} \in \mathbb{C}$ and $\frac{d x_{I}}{x_{I}}=\frac{d x_{i_{1}}}{x_{i_{1}}} \wedge \cdots \wedge \frac{d x_{i_{q}}}{x_{i_{q}}}$.

Proof. Write $\omega=\sum_{i=i_{0}}^{\infty} \omega_{i}$, where the coefficients of $\omega_{i}$ are polynomials of degree $i$ and $\omega_{i_{0}} \neq 0$. If $\varphi_{t}(x)=t \cdot x$ then

$$
\frac{\left(\varphi_{t}\right)^{*} \omega}{t^{i_{0}+q}}=\omega_{i_{0}}+\sum_{i=i_{0}+1}^{\infty} t^{i-t_{0}+q} \omega_{i}
$$

Since for arbitrary $t, \varphi_{t}^{*} \omega$ must be a multiple of $\omega$, after dividing by a suitable formal function we can assume that $\omega$ is homogeneous.

Let $x^{J} d x_{I}$ be a monomial appearing in $\omega$. Suppose $x_{1}^{j_{1}}$ divides $x^{J}$ but $x_{1}^{j_{1}+1}$ does not. Consider the automorphism $\varphi_{t}\left(x_{1}, x_{2}, \ldots, x_{n}\right)=\left(t x_{1}, x_{2}, \ldots, x_{n}\right)$. Then $\varphi_{t}^{*}\left(x^{J} d x_{I}\right)=$ $t^{j_{1}+\epsilon} x^{J} d x_{I}$, where $\epsilon=0$ if $d x_{1}$ does not appear in $d x_{I}$, and $\epsilon=1$ otherwise. If $j_{1}+\epsilon \geq 2$ then $x_{1}$ divides all the other monomials appearing in $\omega$. Thus after division we can assume $j_{1}+\epsilon=1$ and the same will hold true for any other monomial appearing in $\omega$. Repeating the argument for the other coordinate functions clearly yields the assertion of the lemma.

Proposition 6.2. Let $\xi$ be a germ of holomorphic vector field on $\left(\mathbb{C}^{n}, 0\right)$ with a nonresonant singularity at the origin. Suppose $\xi$ is an infinitesimal automorphism of a distribution $\mathcal{D}$ of codimension $q \leq n-2$. Then $\mathcal{D}$ is integrable and the singular set of $\mathcal{D}$ has positive dimension.

Proof. Let $\omega$ be a germ of holomorphic $q$-form, $q=n-p$, defining $\mathcal{D}$, that is, $\mathcal{D}=$ $\left\{v \in T\left(\mathbb{C}^{n}, 0\right) \mid \omega(v)=0\right\}$. For further use let us recall that a $q$-form $\omega$ defines a codimension $q$ distribution if and only if

$$
\left(i_{v} \omega\right) \wedge \omega=0 \quad \text { for every } v \in \bigwedge^{q-1} \mathbb{C}^{n},
$$

and this distribution is integrable if and only if

$$
\left(i_{v} \omega\right) \wedge \omega=\left(i_{v} \omega\right) \wedge d \omega=0 \quad \text { for every } v \in \bigwedge^{q-1} \mathbb{C}^{n}
$$

(see [10]). It follows that integrability is a formal condition, and as such can be verified in an arbitrary formal coordinate system.

Since the origin is a non-resonant singularity for $\xi$, we can choose formal coordinates such that

$$
\xi=\sum_{i=1}^{n} \lambda_{i} x_{i} \partial_{x_{i}}
$$

where $\lambda_{i} \in \mathbb{C}$ are complex numbers. However, we can no longer assume that $\omega$ is a holomorphic $q$-form, but it is certainly a formal $q$-form. 
Since $\xi$ is an infinitesimal automorphism of $\mathcal{D}$, its flow $\varphi_{t}:\left(\mathbb{C}^{n}, 0\right) \rightarrow\left(\mathbb{C}^{n}, 0\right)$ preserves $\omega$. More precisely,

$$
\varphi_{t}^{*} \omega=f(t, x) \omega
$$

for a suitable formal function $f \in \mathbb{C}\left[\left[t, x_{1}, \ldots, x_{n}\right]\right]$.

Consider now the subgroup $G \subset\left(\mathbb{C}^{*}\right)^{n} \subset G L\left(\mathbb{C}^{n}\right)$ defined as

$$
G=\left\{A \in\left(\mathbb{C}^{*}\right)^{n} \mid A^{*} \omega \wedge \omega=0 \text { in } \wedge^{2} \Omega^{q} \otimes \mathbb{C}\left[\left[x_{1}, \ldots, x_{n}\right]\right]\right\},
$$

where $\left(\mathbb{C}^{*}\right)^{n}$ acts on $\left(\mathbb{C}^{n}, 0\right)$ through a diagonal linear map. The flow of $\xi$ determines a non-closed one-parameter subgroup of $H \subset G$. Since $G$ is clearly an algebraic subgroup, it follows that the Zariski closure of $H$ is also contained in $G$. But the dimension of the Zariski closure of $H$ is nothing other than the rank of the $\mathbb{Z}$-module generated by $\lambda_{1}, \ldots, \lambda_{n}$. It follows that $\bar{H}=G=\left(\mathbb{C}^{*}\right)^{n}$.

On the one hand, since $\omega$ induces a distribution, $i_{v} \omega \wedge \omega=0$. On the other hand, Lemma 6.1 implies that $\omega$ is a multiple of a closed $q$-form, and consequently $i_{v} \omega \wedge d \omega$ $=0$. This shows that $\mathcal{D}$ is integrable.

It remains to prove that the singular set of $\mathcal{D}$ has positive dimension. Looking at the expression (6.1) we realize that it must have at least two non-trivial summands. Indeed, if not, $\mathcal{D}$ would be a smooth foliation tangent to $\xi$, which is clearly impossible. Therefore, if $k$ is the cardinality of the set $\bar{I}=\bigcup_{\lambda_{I} \neq 0} I$, where the complex numbers $\lambda_{I}$ are defined by (6.1), then $k>q$. Clearly, the coordinate hyperplanes with index in $\bar{I}$ are invariant under $\mathcal{D}$. Consequently, the intersection of any $q+1$ of these hyperplanes is also invariant under $\mathcal{D}$. Since $\mathcal{D}$ has codimension $q$, this intersection must be contained in the singular locus of $\mathcal{D}$.

Remark 6.3. Proposition 6.2 will be in the proof of the general case of Theorem 1 what Proposition 3.2 is in the proof of the three-dimensional case. The analogy is not perfect as we do not prove here the integrability of multi-distributions as we did there. Anyway, with some extra effort one can also prove the integrability of the multi-distribution. We will not pursue this here as the result above is sufficient for our purposes.

\section{Proof of Theorem 1}

Let $\mathcal{F} \in \mathbb{P} H^{0}\left(X, T X \otimes \mathcal{L}^{\otimes k}\right)$ be a very generic foliation. We can assume, thanks to Theorem 3.5, that $\mathcal{F}$ has isolated singularities, at least one non-resonant singularity, and every leaf of $\mathcal{F}$ is Zariski dense.

Proposition 5.2 implies that $\mathcal{F}$ is tangent to a multi-distribution $\mathcal{D}$. We can assume $\mathcal{D}$ is irreducible without loss of generality.

If $\mathcal{D}$ is locally decomposable around $p$ then Proposition 6.2 implies the existence of a positive-dimensional irreducible component $Z$ of the singular set of $\mathcal{D}$ through $p$. This set is clearly algebraic and invariant under $\mathcal{F}$ since sections of $T \mathcal{F}$ are infinitesimal automorphisms of $\mathcal{D}$. If $\mathcal{D}$ is not locally decomposable at $p$ then there exists a subvariety $Z \subsetneq X$ where $\mathcal{D}$ is not locally decomposable. As above, we conclude that $Z$ is invariant under $\mathcal{F}$.

In both cases, we arrive at a contradiction with Theorem 3.5. 


\section{Bernstein-Lunts Conjecture}

Theorem 1 implies the existence of foliations, on arbitrary projective varieties, with quasiminimal characteristic variety. Moreover, as the conclusion of Theorem 3.5 holds true for any foliation with ample cotangent bundle on $\mathbb{P}^{n}$, the existential part of the BernsteinLunts Conjecture is settled. Nevertheless, there is still a detail to be dealt with in order to prove that a very generic polynomial vector field of degree $d \geq 2$ has quasi-minimal characteristic variety.

\subsection{Projective versus affine degree}

The (projective) degree of a holomorphic foliation $\mathcal{F}$ on $\mathbb{P}^{n}$ is defined as the degree of the tangency divisor of $\mathcal{F}$ with a generic hyperplane $H$. If $\mathcal{F} \in \mathbb{P} H^{0}\left(\mathbb{P}^{n}, \mathcal{O}_{\mathbb{P}}(k)\right)$ then the degree of $\mathcal{F}$ is equal to $k+1$.

If one starts with a polynomial vector field $\xi$ of degree $d$ on $\mathbb{C}^{n}$ then it is natural to extend it to a holomorphic foliation $\mathcal{F}_{\xi}$ on $\mathbb{P}^{n}$ such that $H$ is not contained in the singular set of $\mathcal{F}_{\xi}$. We set the degree of $\xi=\sum a_{i} \partial_{i}$ as the maximal degree of its coefficients $a_{i}$. In general the (projective) degree of $\mathcal{F}_{\xi}$ is at most the (affine) degree of $\xi$. Moreover precisely,

$$
\operatorname{deg}\left(\mathcal{F}_{\xi}\right)= \begin{cases}\operatorname{deg}(\xi) & \text { if } H \text { is invariant under } \mathcal{F}_{\xi}, \\ \operatorname{deg}(\xi)-1 & \text { if } H \text { is not invariant under } \mathcal{F}_{\xi}\end{cases}
$$

If $\mathcal{D}(n, d)$ is the set of polynomial vector fields of degree at most $d$ then the generic element in it extends to a foliation of $\mathbb{P}^{n}$ with singularities of codimension at least two which leaves the hyperplane at infinity invariant (see [12] for a through discussion). In more intrinsic terms, if $T \mathbb{P}^{n}(-\log H)$ denotes the subsheaf of $T \mathbb{P}^{n}$ constituted by germs of vector fields tangent to $H$ then $\mathcal{D}(n, d)$ can be identified with $H^{0}\left(\mathbb{P}^{n}, T \mathbb{P}^{n}(-\log H) \otimes\right.$ $\left.\mathcal{O}_{\mathbb{P}^{n}}(d-1)\right)$. Under this identification the extensions which do not leave the hyperplane at infinity invariant will appear with a divisorial component in their singular set supported there.

\subsection{Relative version of Theorem 3.5}

The proof of Theorem 3.5 can be adapted to prove the following

Theorem 8.1. Let $X$ be a smooth projective variety and $H \subset X$ a smooth hypersurface. Let also $\mathcal{L}$ be an ample line bundle over $X$, and $k \gg 0$ a sufficiently large integer. If $\mathcal{F} \in \mathbb{P} H^{0}\left(X, T X(-\log H) \otimes \mathcal{L}^{\otimes k}\right)$ is a very generic foliation then every leaf of $\mathcal{F}$ not contained in $H$ is Zariski dense.

We will not detail its proof as the case of projective spaces (the one used in the proof of Theorem 2 below) is Theorem 4.2 of [5]. Moreover, there it is proved that it suffices to take $k \geq 1$ when $X=\mathbb{P}^{n}$ and $\mathcal{L}=\mathcal{O}_{\mathbb{P}^{n}}(1)$. 


\subsection{Proof of Theorem 2}

According to Theorem 8.1 the leaves of a very generic vector field of degree $d \geq 2$ are Zariski dense. Also a very generic vector field has at least one non-resonant singularity. Thus we can apply Propositions 5.2 and 6.2 to conclude that the characteristic variety of $\mathcal{F}_{\xi}$ is quasi-minimal.

Acknowledgments. I am grateful to S. C. Countinho for, since 2003, bringing periodically the Bernstein-Lunts conjecture to my attention.

\section{References}

[1] Almeida, L. C. O., Coutinho, S. C.: On homogeneous minimal involutive varieties. LMS J. Comput. Math. 8, 301-315 (2005) Zbl 1111.32031 MR 2193215

[2] Bernstein, J.: Analytic continuation of generalized functions with respect to a parameter. Funktsional. Anal. i Prilozhen. 6, no. 4, 26-40 (1972) (in Russian) Zbl 0282.46038 MR 0320735

[3] Bernstein, J., Lunts, V.: On nonholonomic irreducible $D$-modules. Invent. Math. 94, 223-243 (1988) Zbl 0658.32009 MR 0958832

[4] Cerveau, D.: Feuilletages holomorphes de codimension 1. Réduction des singularités en petites dimensions et applications. In: Dynamique et géométrie complexes, Panor. Synthèses 8, Soc. Math. France, Paris, 11-47 (1999) Zbl 1029.32015 MR 1760842

[5] Coutinho, S. C.: Foliations of multiprojective spaces and a conjecture of Bernstein and Lunts. Trans. Amer. Math. Soc. 363, 2125-2142 (2011) Zbl pre05885463 MR 2746677

[6] Coutinho, S. C., Pereira, J. V.: On the density of algebraic foliations without algebraic invariant sets. J. Reine Angew. Math. 594, 117-135 (2006) Zbl 1116.32023 MR 2248154

[7] Ilyashenko, Y., Yakovenko, S.: Lectures on Analytic Differential Equations. Grad. Stud. Math. 86, Amer. Math. Soc., Providence, RI (2008) Zbl 1186.34001 MR 2363178

[8] Lunts, V.: Algebraic varieties preserved by generic flows. Duke Math. J. 58, 531-554 (1989) Zbl 0696.14009 MR 1016433

[9] McCune, T. C.: A generic cubic surface contains no involutive curve. J. Algebra 235, 36-44 (2001) Zbl 1060.14522 MR 1807653

[10] de Medeiros, A. S.: Singular foliations and differential $p$-forms. Ann. Fac. Sci. Toulouse Math. (6) 9, 451-466 (2000) Zbl 0997.58001 MR 1842027

[11] Stafford, J. T.: Nonholonomic modules over Weyl algebras and enveloping algebras. Invent. Math. 79, 619-638 (1985) Zbl 0558.17011 MR 0782240

[12] Zakeri, S.: Dynamics of singular holomorphic foliations on the complex projective plane. In: Laminations and Foliations in Dynamics, Geometry and Topology, Contemp. Math. 269, Amer. Math. Soc., Providence, RI, 179-233 (2001) Zbl 1193.37066 MR 1810540 